\title{
Design studies for the Phase II upgrade of the CMS Barrel Electromagnetic Calorimeter
}

\section{Toyoko J. Orimoto*}

Northeastern University, on behalf of the CMS Collaboration

E-mail: t.orimoto@northeastern.edu

The High Luminosity LHC (HL-LHC) will provide unprecedented instantaneous and integrated luminosity. The lead tungstate crystals forming the barrel part of the Electromagnetic Calorimeter (ECAL) of the Compact Muon Solenoid (CMS) will still perform well, even after the expected integrated luminosity of $3000 \mathrm{fb}^{-1}$ at the end of HL-LHC. The avalanche photodiodes (APDs) used to detect the scintillation light will also continue to be operational, although there will be some increase in noise due to radiation-induced dark currents. This will be mitigated by reducing the barrel operating temperature during HL-LHC running.

The front-end electronics of the ECAL barrel will be replaced, in order to remove existing constraints on trigger rate and latency and to provide additional capability to fully exploit the higher luminosity delivered by the HL-LHC. New developments in high-speed optical links will allow single-crystal readout at $40 \mathrm{MHz}$ to upgraded off-detector processors, allowing maximum flexibility and enhanced triggering possibilities. Improved algorithms, currently being evaluated in Run II, are being developed to reduce the effects of out-of-time pileup on signal amplitude reconstruction.

The very-front-end system will also be upgraded, to provide improved rejection of anomalous signals in the APDs and to further mitigate the increases in APD noise and pileup. The potential to provide precise timing measurements for high energy signals (with a precision of approximately 20-30 ps) is being explored in the design of the new electronics. This should further mitigate the effects of large event pileup at HL-LHC, where around 200 interactions per $25 \mathrm{~ns}$ bunch crossing are anticipated. The requirements of the new ECAL electronics will be reviewed and the status of design studies and evaluations of demonstrator systems will be provided. Finally, a summary of the expected performance of the upgraded detector during HL-LHC operation will be presented.

38th International Conference on High Energy Physics

3-10 August 2016

Chicago, USA

*Speaker. 


\section{Introduction}

The Compact Muon Solenoid (CMS) Experiment is a general-purpose particle detector experiment at the Large Hadron Collider (LHC) at the CERN laboratory in Geneva, Switzerland [1]. The CMS Experiment was designed to study high energy proton-proton collisions, with the goals of understanding the origin of electroweak symmetry breaking and discovering new physics at the energy frontier. The high resolution CMS electromagnetic calorimeter (ECAL) was a crucial component in the discovery of the Higgs boson, in particular in the $H \rightarrow \gamma \gamma$ and $H \rightarrow Z Z \rightarrow 4 l$ channels. In order to maintain this excellent performance through the high luminosity upgrade of the LHC (HL-LHC), the ECAL barrel will be upgraded, as described below.

\section{CMS ECAL}

The CMS ECAL is a hermetic, homogenous, fine-grained calorimeter consisting of 75848 scintillating lead tungstate crystals, located inside the CMS superconducting solenoid [2]. The ECAL crystals are organized into a central barrel (EB) region, with coverage in pseudorapidity $|\eta|<1.479$, and two endcap regions (EE) with coverage $1.479<|\eta|<3$.0. A preshower detector consisting of two planes of silicon sensors interleaved with a total of $3 X_{0}$ of lead is located in front of EE. In the barrel, the crystals are organized into modules and super-modules of 1700 crystals, and the scintillation light is detected by avalanche photo-diodes (APDs). The ECAL design provides the fast response time, fine granularity, excellent energy resolution and radiation hardness that are required at the LHC. For instance, in the EB, an energy resolution of about $1 \%$ is achieved for unconverted or late-converting photons in the tens of $\mathrm{GeV}$ energy range. The remaining barrel photons have a resolution of about $1.3 \%$ up to a pseudorapidity of $|\eta|=1$, rising to about $2.5 \%$ at $|\eta|=1.4$ [3]. The performance of the ECAL in Run 2 is described in further detail in [4].

A schematic of the current EB readout electronics is depicted in Figure 1a. For each EB crystal, two APDs are mounted on the rear face and connected in parallel to form one readout unit per crystal. The APDs are connected to a passive motherboard, which distributes the high voltage and low voltage and interconnects the APDs to the very-front-end (VFE) cards. Each VFE card has five readout channels made up of a multi-gain pre-amplifier (MGPA) with 43 ns shaping time and a 12-bit analog-to-digital converter (ADC). The VFE cards are capable of operating at a rate of up to $40 \mathrm{MHz}$. The digitized signals from five VFE cards $(5 \times 5$ crystals $)$ form a trigger tower (TT) and are passed to a front-end (FE) card as a single readout unit. The FE cards calculate the trigger primitives from the transverse energy deposited in a TT and a single bit to qualify the energy deposit. The trigger primitives are transmitted optically off-detector to a trigger concentrator card in the underground service cavern. In addition, the FE cards also contain the digital latency buffer and primary event buffer. The per-crystal information is buffered in the FE for transmission to the data concentrator card. Only channels above an energy threshold, associated to a cluster of deposits, are read out. The VFE and FE were fabricated with $0.25 \mu \mathrm{m}$ radiation-hard CMOS technology. All boards were tested to resist the radiation environment for the entire duration of the LHC.

\section{High-Luminosity LHC}

The LHC was designed to provide $500 \mathrm{fb}^{-1}$ of $13 \mathrm{TeV}$ proton collisions, over 10 years, at a 


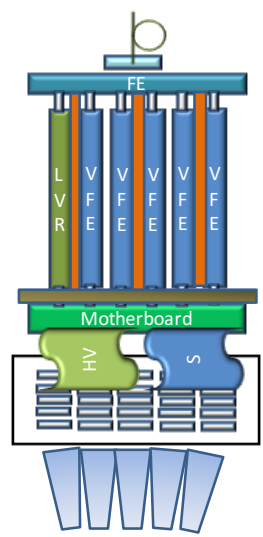

(a)

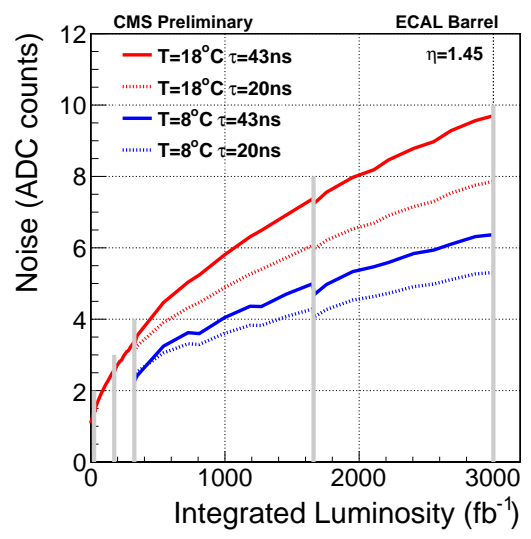

(b)

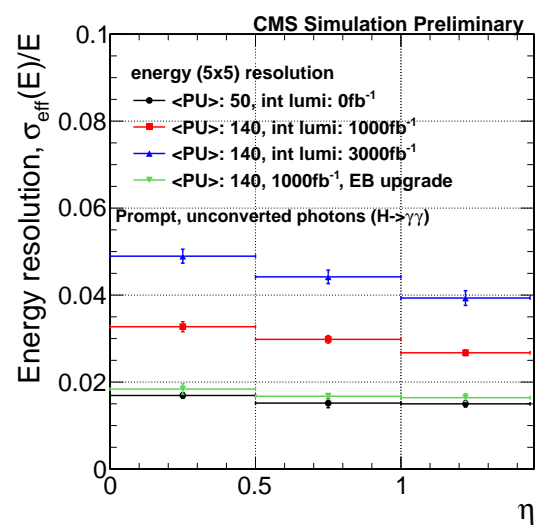

(c)

Figure 1: (a) Schematic of CMS ECAL readout electronics, depicting crystals (at the bottom), the motherboard, 5 VFE boards, and $1 \mathrm{FE}$ board. (b) Expected noise level in EB versus integrated luminosity at et $a=1.45$ for operating temperatures of $18{ }^{\circ} \mathrm{C}$ (red curves) or at $8{ }^{\circ} \mathrm{C}$ (blue curves), with the present electronics (continuous line, shaping time $\tau=43 \mathrm{~ns}$ ), or the upgraded electronics (dotted line, $\tau=20 \mathrm{~ns}$ ). (c) Energy resolution $\sigma_{\mathrm{eff}}(E) / E$ for photons from simulated $H \rightarrow \gamma \gamma$ events, for different integrated luminosities and pileup, showing the resolution improvement provided by the upgrade (EB operated at $8{ }^{\circ} \mathrm{C}$ and $\tau=20 \mathrm{~ns}$ ).

peak instantaneous luminosity of $1 \times 10^{34} \mathrm{~cm}^{-2} \mathrm{~s}^{-1}$. Beyond that point, the HL-LHC will provide unprecedented instantaneous $\left(5 \times 10^{34} \mathrm{~cm}^{-2} \mathrm{~s}^{-1}\right)$ and integrated luminosity $\left(3000 \mathrm{fb}^{-1}\right)$ until 2035 [5]. The physics goals for the HL-LHC include precise measurements of the Higgs boson couplings and studies of rare SM processes, both of which may provide clues about the nature of potential new physics, as well as direct searches for new phenomena. An important benchmark for the HL-LHC will be the measurement of the Higgs self-coupling through di-Higgs production, for which the final state of two b-quarks and two photons may be the most sensitive channel. The continuing good performance of the ECAL at the HL-LHC will be crucial for this measurement.

\section{ECAL Barrel Upgrade}

The CMS ECAL will face a challenging environment at the HL-LHC: higher event pile-up (PU) from multiple interactions per bunch crossing (average PU will increase from 50 to 140), higher radiation levels $(\times 6)$ for the crystals and photo-detectors, and a higher rate of anomalous signals in the APDs. To mitigate these challenges and maintain the excellent physics performance of the detector, an upgrade to the ECAL is planned. The EE will have to be replaced entirely, due to irrecoverable radiation damage to the EE crystals and thus significant degradation in performance. The EE upgrade is outside the scope of this report but has been described elsewhere [5].

The primary motivations for an upgrade of the ECAL barrel electronics are the latency and bandwidth requirements of the upgraded CMS trigger system, which will be improved in order to accommodate the high event rates at the HL-LHC. The EB crystals, exposed to less radiation in the central region, will continue to perform well throughout the HL-LHC and will not be replaced. 
However, the EB readout electronics must be upgraded in order to accommodate the increase in Level-1 (L1) trigger rate (100 kHz at LHC compared to $750 \mathrm{kHz}$ at HL-LHC) and latency ( $5 \mu \mathrm{s}$ at LHC compared to $12.5 \mu \mathrm{s}$ at HL-LHC) planned for the upgraded CMS trigger system [5]. The upgrade of the EB readout electronics will necessitate the removal, refurbishment, reinstallation and recommissioning of the $36 \mathrm{~EB}$ supermodules during the Phase II upgrade program of CMS. The crystals, APDs, motherboards, and overall mechanical structure of the EB will remain the same. Additional improvements that are foreseen during the EB upgrade include: reduction of the increasing APD noise due to dark current; improvements in anomalous signal rejection and PU mitigation; precision timing for vertex determination and PU mitigation.

\subsection{APD noise}

Although the APDs are expected to remain operational through HL-LHC, the APD dark current increases with integrated luminosity, resulting in higher noise levels. We expect that the APD noise will increase from $40 \mathrm{MeV}$ per channel during the initial commissioning period of ECAL to $400 \mathrm{MeV}$ per channel at the end of the HL-LHC. To mitigate this effect, the EB will be operated at a lower temperature- $8{ }^{\circ} \mathrm{C}$ compared to the current operating temperature of $18{ }^{\circ} \mathrm{C}$. Figure $1 \mathrm{~b}$ shows the reduction in dark current induced APD noise by reducing the $\mathrm{EB}$ operating temperature: $\sim 6$ ADC counts $(\sim 240 \mathrm{MeV})$ for $8{ }^{\circ} \mathrm{C}$ vs $\sim 10$ ADC counts $(\sim 400 \mathrm{MeV})$ for $18{ }^{\circ} \mathrm{C}$ at the end of the HL-LHC. Figure $1 \mathrm{~b}$ also illustrates a further reduction in noise by decreasing the pulse shaping time in the VFE from 43 ns to $20 \mathrm{~ns}$. Reducing the EB operational temperature will require careful planning of services, as well as optimization of thermal insulation to avoid condensation. Figure $1 \mathrm{c}$ shows the improvement in energy resolution provided by operating the EB at $8{ }^{\circ} \mathrm{C}$ and a shaping time of $\tau=20 \mathrm{~ns}$, measured with simulated $H \rightarrow \gamma \gamma$ events at the HL-LHC.

\subsection{Anomalous signal rejection}

Anomalous signals ("spikes"), consisting of isolated large signals with equivalent energies that can exceed $100 \mathrm{GeV}$, have been observed in EB during $p p$ collisions [6]. The spikes arise when particles from $p p$ collisions directly strike the APDs and very occasionally interact to produce secondary particles that cause large anomalous signals through direct ionization. The spikes have been observed at a rate that is proportional to the collision rate, and as such pose a potential problem for triggering at high luminosities. The spikes can be distinguished from true scintillation signals from electromagnetic (EM) showers using the topology of the deposits in EB, since the spikes occur in a single channel. In addition, the pulse shape of the spikes is also different from EM showers, arriving earlier in time and with a shorter pulse since the spike signals do not include the response time of the scintillation light. Currently, spikes are rejected at L1 using a coarse topological algorithm, since single crystal granularity is not yet available at L1. Without an upgrade, the efficiency of these rejection algorithms will degrade to unacceptable levels at the HL-LHC, due to the higher collision rate and higher levels of noise and PU. However, the upgraded electronics will allow us to apply more sophisticated filtering algorithms in the VFE since single crystal data will be made available at the trigger level, as described below. In addition, decreasing the VFE shaping time will enable the use of the difference in pulse shape to further improve the rejection efficiency of spikes. Figure 2a compares the pulse shapes for a spike signal and a true scintillation signal from an EM shower. 


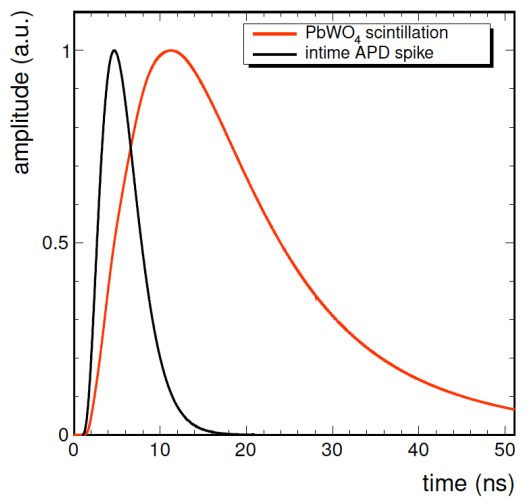

(a)

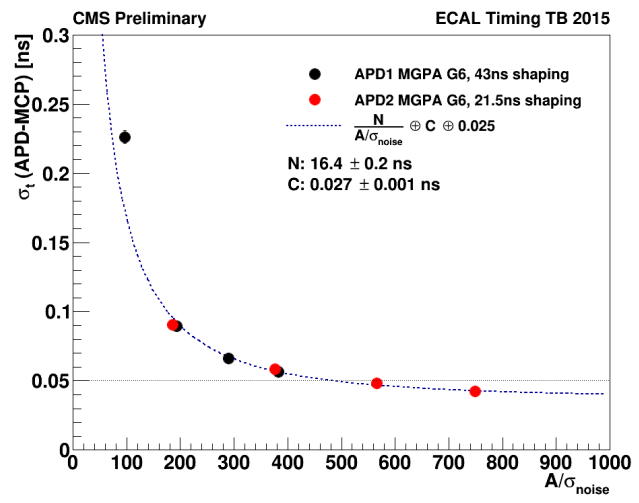

(b)

Figure 2: (a) Pulse shapes for a spike (black) and a scintillation signal from an EM shower (red) from simulation. (b) EB timing resolution as a function of the normalized amplitude $(A / \sigma)$, for pulse shaping times of $\tau=43 \mathrm{~ns}$ and $20 \mathrm{~ns}$.

\subsection{Precision timing}

The vertex resolution for $H \rightarrow \gamma \gamma$ events will degrade in the high PU environment of the HL-LHC, but can be restored by utilizing precision timing. Precision timing can also be used to improve the subtraction of neutral energy from PU that enters EM clusters and jets, and to improve the identification of PU jets. The intrinsic timing resolution of the EB was measured to be $<30$ ps at a CERN test beam, as depicted in Figure 2b. The upgraded EB system will be designed to approach the instrinsic $30 \mathrm{ps}$ timing precision of the crystals and APDs for high energy EM signals.

\subsection{EB electronics upgrade}

The upgrade of the EB electronics will entail replacement of the on-detector VFE and FE cards, along with the associated low-voltage distribution system and optical links. The upgrade will be optimized such that the basic performance specifications of the current system will be maintained. The VFE will maintain a similar purpose, but the shaping time and sampling rate will be optimized for HL-LHC conditions. A reduction in shaping time (for example, from 43 ns to $20 \mathrm{~ns}$ ) in the VFE will significantly reduce the effective noise from PU and electronics noise, while also allowing for better discrimination spike rejection. Shorter shaping time will also improve the determination of the pulse arrival time for spike rejection, pileup suppression, and vertex association. The pulse shaping and preamplifier ASIC options that are currently being investigated use a trans-impedance amplifier (TIA) or an iteration of the current architecture (MGPA CR-RC) as an alternative. For the digitizer, multi-channel ADCs with 12 bit resolution and sampling rates up to $160 \mathrm{MHz}$ are being considered. VFE prototype boards have been undergoing lab and test beam measurements, in parallel with simulation studies to optimize the shaping time and sampling rate. The new ASICs for the VFE and FE will require a different bias voltage than what is currently supplied, necessitating an upgrade for the low-voltage regulator card (LVR). For the control and distribution of the clock, the trigger commands, and FE and VFE settings and configurations, the Gigabit Transceiver ASIC (GBTX) control bus will be used to serve groups of 25 crystals, simplifying the current system which uses a specific control board. 
The requirements for the FE and off-detector electronics are to read data from all crystals, with a longer data pipeline for the increased trigger latency. As such, the FE card will become a pipeline, transferring most of the processing to the off-detector electronics. Radiation-hard $10 \mathrm{~Gb} / \mathrm{s}$ optical links, currently under development at CERN, will allow the transmission all channels from the FE to the off-detector electronics, with no data reduction performed in the VFE or FE and utilizing the same stream for trigger and data. An FE demonstrator with $5 \mathrm{~Gb} / \mathrm{s}$ links is currently undergoing lab measurements. The off-detector electronics will also be upgraded to accept the higher rates, including single crystal readout. In addition, the generation of trigger primitives will be shifted off-detector in the service cavern. As such, the upgraded processors need not be radiation-hard and commercially available FPGAs can be utilized. The L1 trigger pipeline will also be moved off-detector, allowing for an arbitrary trigger latency. Moreover, the trigger, data and controls may be grouped in single card in the off-detector electronics. An additional benefit of this new readout scheme is the ability to apply more advanced topological filtering to reject spikes at the trigger level, similar to the filtering methods that are currently applied offline.

\section{Conclusions}

The CMS ECAL barrel crystals, APDs, and electronics have performed well at the LHC. However, the challenging conditions of the HL-LHC necessitate an EB upgrade, which will entail full refurbishment of the EB readout electronics to accommodate the higher trigger bandwidth and latency as well as to mitigate the increase in spikes and PU. In addition, a lowering of the EB operating temperature will mitigate the increase in dark current induced APD noise. Precision timing is also desired to improve vertex determination and PU mitigation. The Phase-II upgrade will allow us to maintain the excellent energy resolution of the EB through the end of the HL-LHC.

\section{References}

[1] S. Chatrchyan et al. [CMS Collaboration], "The CMS experiment at the CERN LHC," JINST 3, S08004 (2008).

[2] S. Chatrchyan et al. [CMS Collaboration], "Performance and Operation of the CMS Electromagnetic Calorimeter," JINST 5, T03010 (2010).

[3] V. Khachatryan et al. [CMS Collaboration], "Performance of Photon Reconstruction and Identification with the CMS Detector in Proton-Proton Collisions at sqrt(s) $=8$ TeV," JINST 10, 08, P08010 (2015).

[4] M. Sun (on behalf of the CMS Collaboration), "Achieving the optimal performance of the CMS ECAL in Run II," ICHEP 2016, these proceedings.

[5] CMS Collaboration, "Technical Proposal for the Phase-II Upgrade of the CMS Detector," CERN-LHCC-2015-010.

[6] D. A. Petyt [CMS Collaboration], "Mitigation of anomalous APD signals in the CMS electromagnetic calorimeter," J. Phys. Conf. Ser. 404, 012043 (2012). 